\title{
A determinantal representation for derangement numbers
}

\author{
Feng $\mathbf{Q i}^{1,2,3, *}$ \\ ${ }^{1}$ Institute of Mathematics, Henan Polytechnic University, Jiaozuo City, Henan Province, 454010, China \\ ${ }^{2}$ College of Mathematics, Inner Mongolia University for Nationalities, Tongliao City, Inner Mongolia Autonomous Region, 028043, China \\ ${ }^{3}$ Department of Mathematics, College of Science, Tianjin Polytechnic University, Tianjin City, 300387, China \\ *Corresponding author E-mail: qifeng618@gmail.com
}

\begin{abstract}
In the note, the author finds a representation for derangement numbers in terms of a tridiagonal determinant whose elements are the first few natural numbers.
\end{abstract}

Keywords: derangement number; determinantal representation; tridiagonal determinant.

In combinatorics, a derangement is a permutation of the elements of a set, such that no element appears in its original position. The number of derangements of a set of size $n$ is called derangement number and sometimes denoted by $! n$. The first ten derangement numbers $! n$ for $0 \leq n \leq 9$ are

$1, \quad 0, \quad 1, \quad 2, \quad 9, \quad 44, \quad 265, \quad 1854, \quad 14833,133496$.

We now discover that derangement numbers $n$ can be beautifully expressed as a certain explicitly written down tridiagonal determinant. To the best of our knowledge, we have not seen such a representation in the context earlier.

Theorem 1. For $n \in\{0\} \cup \mathbb{N}$, derangement numbers $! n$ can be expressed by a tridiagonal $(n+1) \times(n+1)$ determinant

$$
\begin{aligned}
! n=-\left|\begin{array}{cccccccc}
-1 & 1 & 0 & 0 & \ldots & 0 & 0 & 0 \\
0 & 0 & 1 & 0 & \ldots & 0 & 0 & 0 \\
0 & -1 & 1 & 1 & \ldots & 0 & 0 & 0 \\
0 & 0 & -2 & 2 & \ldots & 0 & 0 & 0 \\
0 & 0 & 0 & -3 & \ldots & 0 & 0 & 0 \\
\vdots & \vdots & \vdots & \vdots & \ddots & \vdots & \vdots & \vdots \\
0 & 0 & 0 & 0 & \ldots & n-3 & 1 & 0 \\
0 & 0 & 0 & 0 & \ldots & -(n-2) & n-2 & 1 \\
0 & 0 & 0 & 0 & \ldots & 0 & -(n-1) & n-1
\end{array}\right| \\
=-\left|e_{i j}\right|_{(n+1) \times(n+1),}
\end{aligned}
$$

where

$e_{i j}= \begin{cases}1, & i-j=-1, \\ i-2, & i-j=0, \\ 2-i, & i-j=1, \\ 0, & i-j \neq 0, \pm 1 .\end{cases}$

Proof. Once we write down the determinant, the proof of Theorem 1 can be made into a single line! Indeed, if the determinant written down in Theorem 1 is denoted by $a_{n}$, then an induction immediately gives $a_{n+1}=n\left(a_{n}+a_{n-1}\right)$. This clearly produces derangement numbers $! n$ which are determined by this recursion. Once discovered, the proof is just a single line.

Remark 1 . Recently, an alternative, although slightly complicated, proof of Theorem 1 was supplied in [1].

\section{References}

[1] F. Qi, J.-L. Wang, and B.-N. Guo, A recovery of two determinantal representations for derangement numbers, Cogent Math. (2016), in press; Available online at http://dx.doi .org/10.1080/23311835. 2016.1232878. 